\title{
The S-Kernel and a Symmetry Measure Based on Correlation
}

\author{
Bertrand Zavidovique $^{1}$ and Vito Di Gesù ${ }^{1,2}$ \\ 1 IEF, University of Paris XI, ORSAY, France \\ 2 DMA, Università di Palermo, Italy \\ digesu@math.unipa.it, zavido@ief.u-psud.fr
}

\begin{abstract}
Symmetry is an important feature in vision. Several detectors or transforms have been proposed. In this paper we concentrate on a measure of symmetry. Given a transform $S$, the kernel $S K$ of a pattern is defined as the maximal included symmetric sub-set of this pattern. The maximum being taken over all directions, the problem arises to know which center to use. Then the optimal direction triggers the shift problem too. We prove that, in any direction, the optimal axis corresponds to the maximal correlation of a pattern with its flipped version. That leads to an efficient algorithm. As for the measure we compute a modified difference between respective surfaces of a pattern and its kernel. A series of experiments supports actual algorithm validation.
\end{abstract}

\section{Introduction}

This paper deals with measuring a degree of symmetry of $2 D$ subsets of pictures. It helps extracting objects. Symmetry is a prevalent feature in human perception. For instance the human face or body is approximately symmetric that is exploited to assist in face recognition and detection. Psychologists of the Gestalt school have assigned a relevant role to symmetry in attentive mechanism both in visual and auditory systems [1,15. From the survey by Zabrodsky [13, we stress upon results corroborating our own findings in the machine domain: - saliency of vertical symmetry provided mental rotation : detections are in the order vertical, horizontal, bent and then rotational symmetry; - parts near the axis contribute more to symmetry than further parts near edges, themselves more critical than regions in between.

The concept of symmetry is important in machine vision too as confirmed by an extensive literature: a recent quite interesting survey is [2]. Models of symmetry suffer three drawbacks: - $\mathbf{d}_{\mathbf{1}}$ - edges mainly support symmetry detection; - $\mathbf{d}_{\mathbf{2}}$ - perfect symmetry is targeted; - $\mathbf{d}_{\mathbf{3}}$ - the center of mass is assumed to be the focus of attention

Similar difficulties have been long solved for edges, regions or motion in actually measuring the phenomenon - edginess, uniformity, set-direction - to decide after the measure rather than using a strict distance. We addressed $\mathbf{d}_{\mathbf{1}}$ in [14] by defining iterative transforms as the IOT that better account for the 
inner object. In the present paper we tackle all $\mathbf{d}_{\mathbf{i}}$-difficulties together to optimally solve the problem. The bibliography of section 2 suggests tools. In section 3 , we introduce the notion of a "kernel" that stems logically from IOT through a classical gauge in functional analysis. In section 4, preliminary study of the main parameter - the optimal axis to spot - leads to an ultimate detection algorithm based on correlation, and a general enough symmetry measure is derived. A series of experiments in section 5, on both binary and grey scaled pictures, allow to evaluate the technique, to check its sensitivity to the center position and the validity of the degree of symmetry. Short discussion and further comments conclude the paper.

\section{State of the Art}

The use of gray level information was firstly investigated in [6], where the symmetry descriptor is based on a cross correlation of gray levels. In [9], the search for symmetries evaluates the axial moment of a body around its center of gravity. Applied at a local level this descriptor defines the Discrete Symmetry Transform $(D S T)$. In [7, local reflectional symmetry is computed in convolving with the first and second derivative of Gaussians. Each point gets both a symmetry "measure" and an axis orientation. Shen [17] or DuBuff [18] use complex moments with Fourier or Gabor image approximation. It implies axes to pass through the center of mass, and moments are not invariant to affine transforms.

In [8, authors introduce several descriptors from Marola's one extended to finite supports and varying scales based on the Radon and Fourier transforms. Scale dependency is claimed to detect global symmetries without any prior segmentation. The global optimization is then implemented by a probabilistic genetic algorithm for speedup. Likewise, Shen and al. 12 detect symmetry in seeking out the lack of it. The asymmetric term of their measure (energy) is null for any pattern invariant through horizontal reflection, whence minimizing that term over the image. In [11, a multi-scale (see also [10]) vector potential is constructed from the gradient field of filtered images. Edge and symmetry lines are extracted through a vector field (i.e. curl of the vector potential): symmetry axes are where the curl of the vector vanishes and edges are where the divergence of the potential vanishes. Most described methods so far provide symmetry descriptors to compute measures from. Others aim at straight symmetry measures. Comparing for instance Cross's and Yeshurun's, Yeshurun and al. [22] build on the Blum-Asada vein [3], but in quantifying a potential for every pixel to be centre of symmetry based on pairs of edge points tentatively symmetric from their respective gradient vectors. A degree of symmetry is assigned to every pair within a given pixel neighborhood and a weighted combination of these makes the pixel potential, whose local maxima provide a measure depending on both intensity and shape. The technique further extends to textures [23]. The review of preceding works points out that: 1) comparing a pattern with its transformed version, for invariance, can prevent from imposing the centroid as the a priori focus of interest; 2) introducing true measures supports more abstract 
versions of distances, founding approximate comparison; 3) sets which measures apply on may be "sets of pixels or vectors" (shapes) or "sets of patterns" (inclass transforms): in either case "set operations", as Minkowski's ones, are worth considered. They do not limit to contours and bridge logic with geometry.

Three more works fit very well the algorithmic line above and are the closest to ours, making clear the main contributions of this paper. In [19] the authors correlate the image with its transformed version to by-pass the centroid. But they do that on the inner product of (gaussian) gradients, hence on edges. R. Owens 20] searches explicitly for a measure of symmetry to indicate approximate bilateral symmetry of an isolated object. But she defines tentative symmetries from the principal axes of inertia, whence the centroid again, before to compute the sum of absolute differences of grey levels in symmetric pairs over the object, normalized by their maximum. Note that, although it is not mentioned, such a measure amounts to a slightly modified $\mathrm{L}_{1}$-difference between the object and a maximal-for-inclusion symmetric version of it in the given direction. Kazhdan et al. 21] target true visualization of symmetry over every point of a pattern. They use explicitly the same idea of a difference $\left(\mathrm{L}_{2}\right.$ in their case) between the image and its closest symmetric version (the average of the picture and its transform). But they need a measure that integrates all reflective invariance about a bundle of straight lines (or planes in 3-D). It is robust to noise and suitable for object matching, yet a center is necessary to this representation. The representation plots for every direction the measure of symmetry about the normal plane passing through the center of mass. Note that its local maxima point out potential pattern symmetries.

\section{The New Symmetry Measure}

\subsection{Symmetry Indicators (IOT)}

In [14] we defined IOT that is a map product of iterated morphological erosion and symmetry detection.

Definition 1. The Symmetry Transform, $S$, on a continuous object $X \subset R^{2}$ is given by:

$$
S_{\alpha}(\mathrm{X})=\int_{X} m(x) \times \rho^{2}(x, r(\alpha)) d x \quad \text { for } \quad \alpha \in[0, \pi[
$$

where, $r(\alpha)$ is the straight line with slope $\alpha$ passing through the center of gravity of the object $X, m(x)$ is the mass of the object in $x \in X$, and $\rho$ is a distance function of $x$ from the straight line.

Definition 2. The Iterated Object Transform, IOT, is given by:

$$
\begin{aligned}
& \operatorname{IOT}_{\alpha, 1}(X)=S_{\alpha}(X) \\
& \operatorname{IOT}_{\alpha, n}(X)=S_{\alpha}\left[(\mathrm{E})^{n-1}(X)\right] \quad \text { for } n>1
\end{aligned}
$$


$(E)^{n}$ stands for the morphological erosion by the unit sphere (or any other suitable structuring element would any suitable a priori information be available), iterated $n$ times.

The number of iterations depends on the image size and on the gray level distribution. The $S$ transform is thus computed on progressively shrunk versions of the binary input image or on steadily intensity reduced versions of the gray level input image, until some predefined decrease or a minimum of intensity is reached. The iterated elongation, $\eta_{n}(X)$, is defined as:

$$
\eta_{n}(X)=\frac{\min _{\alpha \in[0, \pi[}\left\{\operatorname{IOT}_{\alpha, n}(X)\right\}}{\max _{\alpha \in[0, \pi[}\left\{\operatorname{IOT}_{\alpha, n}(X)\right\}}
$$

It represents dynamic changes of $X$ shapes indicators versus $n$. Since in most cases, $\eta$ curves become flat or show some other type of constancy after a certain number of erosions, it was conjectured that any pattern larger than the structuring element would have a symmetric kernel that IOT reveals (at least one pixel remains after erosion to meet the definition). Let us call IOTK this pattern. The intuitive idea here is that the closer the kernel to the pattern the more symmetric pattern, although it is easy to design examples (see Figure 10) where the IOTK is as "far" as wanted from the pattern.

Remark 1: when it proves necessary, this included version of the kernel could be balanced by the including version obtained by dilation. Then the question arises to define the kernel more formally.

\subsection{Definition of the Kernel}

Following commonly used gauges in functional analysis, a possible first answer with a flavor of optimality would be maximal included symmetric pattern resp. minimal including symmetric pattern.

Definition 3. The $S$-kernel of the pattern $P-S K(P)$ - is the maximal for inclusion symmetric subset of $P$. Let us assume we applied the IOT and found a stable pattern after multiple erosion, like the dark rectangle in the Figure 1,

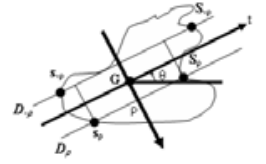

(a)

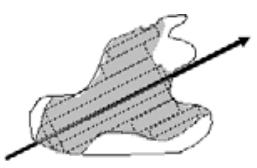

(b)

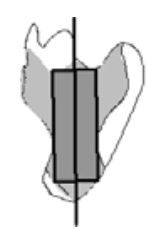

(c)

Fig. 1. (a) Sketch of the kernel detection algorithm; (b) the kernel of the pattern in (a); (c) expanding the IOTK of the pattern in (a) into the kernel 


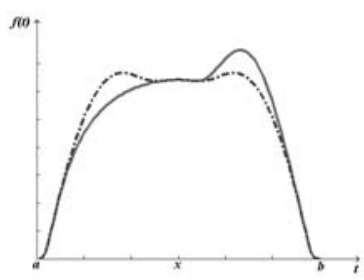

(a)

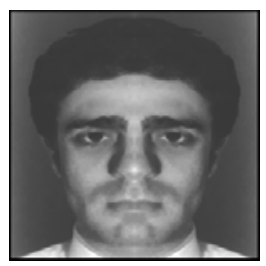

(b)

Fig. 2. (a) Searching for the symmetry axis of a function $y=f(t)$ (plain line): its symmetric version around $x, S_{f}^{x}(t)$ (dotted line), and (b) the best symmetric version of the face Figure $3+1 \mathrm{~b}$

(meaning after that, erosion will confirm symmetry and $\eta$ remains constant). Starting from there we expand the pattern and mark progressively every where the expansion intersects the border, together with the symmetric pixel wrt. the axis. Every marked pixel is not expanded further. That way the kernel should be obtained again, provided the center of mass be stable. That makes the link between the latter kernel and former indicators as IOTK.

Remark 2: the center of mass likely varies from the kernel to the pattern. This introduces an additional question: how to define the likely symmetry axis where to compute the kernel from?

For instance, let be $\mu=\operatorname{argMaxSymmetry}(p t r n)$. How does $K_{\mu}(p t r n)$ compare with $K($ ptrn $)$ ? How do their respective Symmetry relate? In most cases $K_{\mu}(p t r n)$ should be a good enough gauge of $K(p t r n)$, or the difference between them will be most indicative. The following section is entirely devoted to answering that. All complementary tests will result into or from experiments described in section 5 .

\section{Formal Setting and an Algorithm}

The frame of the present study is not mandatorily that of an isolated pattern any more. Considering operators to be used in the end, correlation, our conjecture is rather of an attention focusing process based on symmetry. Introducing the latter notion of interest implies that all non interesting regions around be faded to zero, and that the pattern scan be started for instance at the very left of it. See below the end of 4 and results in 5 for an illustration. Hence, the pattern $f(x)$ gets a bounded support and the origin of the $\mathrm{x}$-axis can be where the interest starts.

\subsection{Optimization of the Center Position}

Let be (Figure 2): $S_{f}^{x}(t)=\frac{f(x+t)+f(x-t)}{2}$ the symmetric version of $f$ with respect to $x$. For the $L_{2}$-norm, the best axis $x^{*}$ corresponds to: 


$$
S_{x *}(f)=\min _{x} \int_{a}^{b}[f(x+t)-f(x-t)]^{2} d t
$$

Considering the above general frame, $a$ can be set to 0 without any loss in generality and support of $f$ is included in that of $S_{f}^{x} . f$ is assumed derivable then bounded integrable. It comes:

$$
\frac{d}{d x} S_{x}(f)=\int_{0}^{b} \frac{d}{d x}[f(x+t)-f(x-t)]^{2} d t
$$

As $f(x)=0$ for $x<0$ and $x>b$,

$$
\frac{d}{d x} S_{x}(f)=2\left(\int_{0}^{b} f(x+t) \times f^{\prime}(x-t) d t-\int_{0}^{b} f^{\prime}(x+t) \times f(x-t) d t\right)
$$

with $x+t=u($ resp. $x-t=u)$ then $x-t=2 x-u($ resp. $x+t=2 x-u)$

$$
\int_{0}^{b} f^{\prime}(x+t) \times f(x-t) d t=\int_{x}^{b+x} f(2 x-u) \times f^{\prime}(u) d u
$$

$\left(\right.$ resp. $\left.\left.\int_{0}^{b} f(x+t) \times f^{\prime}(x-t) d t=\int_{x-b}^{x} f(2 x-u) \times f^{\prime}(u) d u\right)\right)$

$f(t)$ and $f^{\prime}(t)$ being null for $t<0$, it comes in all cases:

$\left.\int_{x-b}^{x+b} f(2 x-u) \times f^{\prime}(u) d u=\int_{0}^{2 x} f(2 x-u) \times f^{\prime}(u) d u=f \otimes f^{\prime}(2 x)\right)=\frac{d}{d x}(f \otimes f)$

with $\otimes$ the convolution product.

\subsection{Correlation and Algorithm}

Eventually $S_{x^{*}}(f)$ corresponds to: $(f \otimes f)$ maximal or equivalently to:

$\int_{\sup (0,2 x-b)}^{\inf (2 x, b)} f(2 x-u) \times f^{\prime}(u) d u=0$ whichever is easier to compute.

One gets yet another algorithm: find the maximum correlation of the picture in a given direction (i.e. over all translations in this direction) with its mirror symmetric in that direction (i.e. scanned right to left). Considering existing efficient algorithms for image geometric transforms (eg. cordic), rotations to span directions can then be performed on the image before scans and correlation: approximations need to be checked for the error introduced remain acceptable (comparable to the one from sampled angles and discrete straight lines).

Remark 2: Note that if the image is tiled adapted to the predicted size of potential symmetric objects, one can derive an efficient focusing process.

It is now obvious that considering the center of mass $G$ for all potential symmetry axes to go through amounts to an approximation of $f$ by the square term of its Taylor expansion, since it is defined by:

$$
X_{G} / \int_{0}^{b}(X-u) f(u) d u=\frac{1}{2} \int_{0}^{b} u^{2} f^{\prime}(X-u) d u=0
$$


that is different in general from:

$$
x^{*} / \int_{\sup (0,2 x-b)}^{i n f(2 x, b)} f(u) \times f^{\prime}(2 x-u) d u=0
$$

As for extending the result in 2 or $3-\mathrm{D}$, formulas are identical for $f(\underline{t})$ with $\underline{t}=(t, s)$ since both derivation and convolution are linear. Actually, one considers $S_{\underline{x} *}(f)=\min _{\underline{x}} \iint_{D}[f(\underline{x}+\underline{t})-f(\underline{x}-\underline{t})]^{2} d t . d s$ with $\underline{x}=(x, 0)$ and $D$ bounded by $\phi(t, s)=0$ determined otherwise or the rectangle frontier of the picture or part of it. It leads to the same formal result since derivation deals with the single variable $\mathrm{x}$ and that makes the case for grey scaled images.

Remark 3: The latter formal setting confirms that using the projection (Radon transform) to extract symmetries from provides necessary conditions only since the result of permuting integrals in $\frac{d}{d x} S_{\underline{x} *}(f)$ is not guaranteed. In the discrete case, it is obvious how to build examples in shuffling column's pixels to get a non symmetric pattern from a symmetric one, still conserving the projection. Even if nature and noise make this type of ambiguities quite rare, in case of multiple symmetries one can be transformed into another by such permutation.

Remark 4: In cases where rotational symmetry would be explicitly looked for, polar coordinates can be used. The same formal result holds too:

$$
S_{\psi}(f)=\int_{0}^{2 \pi} \int_{0}^{\varphi(\theta)}[f(\rho, \theta)-f(\rho, \theta+\psi)]^{2} \rho . d \rho d \theta
$$

since $\rho$ is positive:

$f(\rho, \theta)=g\left(\rho^{2}, \theta\right)=g(u, \theta) \Longrightarrow S_{\psi}(f)=\int_{0}^{2 \pi} \int_{0}^{\sqrt{\varphi(\theta)}}[g(u, \theta)-f(u, \theta+\psi)]^{2} d u d \theta$

It leads again to the same computation except the pole (center of rotation) is assumed to be known, limiting the interest of the result.

\subsection{Symmetry Measure}

A preliminary simplifying assumption is made here: while the kernel was exhibited, the picture is considered to have been binarized or at least the pattern was cropped if it was not before. So, in order to test the proposed algorithm we compute a measure of symmetry classically defined as:

$$
\lambda_{1}=1-\frac{\operatorname{Area}(D)}{\operatorname{Area}(A)}
$$

with $A$, the pattern or a binding sub-picture, $B$, its kernel, and $\operatorname{Area}(D)=$ Area $(A-B)$. Provided suitable binarization or windowing, it remains a robust first approximation where $\lambda_{1}=1$ if $\operatorname{Area}(B)=\operatorname{Area}(A)$. See results in 5.1 . 


\section{Experimental Results and Further Comments}

In this section we show some results of the application of the S-kernel algorithm $(S K A)$ to synthetic and real images. The purpose of experiments can be summarized as follows: 1) validate the ability of the proposed method in measuring the degree of symmetry, by comparing $\mid \eta(S K($ ptrn $))-\eta($ ptrn $) \mid$ with $\lambda$, and $\eta(I O T K)$ with $\eta(S K(p t r n)) ; 2)$ compute the kernel by correlation and compare with IOTK ( from the algorithm in [14]); 3) compare the axis position obtained by correlation with the best center position obtained after IOTK;4) check ability of the algorithm to support attention focusing from symmetry. All

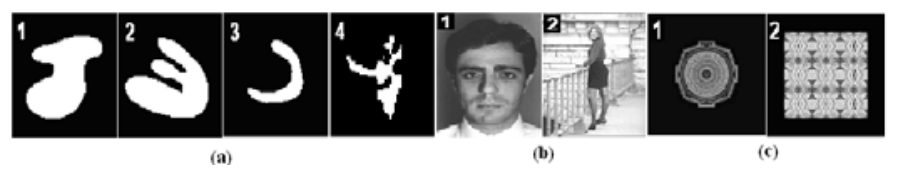

Fig. 3. Sample gallery of images used for experiments: (a) binary; (b) gray level; (c) textured

experiments are parameterized by direction and run on both binary and gray level images (see Figure 3).

\subsection{Evaluating the Correlation Algorithm}

Figures 4] show examples of the similarity measures computed after correlation, $\rho$ and the similarity $\lambda$ against the direction $\alpha$. It is interesting that $S K A$ is able to grasp the circularity of the images $1 c$ and the four axes of symmetry of the image 2c.

Table 1 reports the results for all images in Figure 3. $\rho\left(\rho_{\max }\right)$ indicates the object direction $\alpha$.

We tested the robustness of $S K A$ by rotating the images of $45^{\circ}$ and the results of the computation were $\rho=0.89, \alpha=45.00^{\circ}$ for the image $1 \mathrm{~b}$, and $\rho=0.88, \alpha=135.00^{\circ}$ for the image $2 \mathrm{~b}$.

The direction of images $1 \mathrm{a}, 2 \mathrm{a}$, and $3 \mathrm{a}$ is close to human perceive, the long nose of the sketched profile in image 4 a forces the algorithm to an "horizontal" direction. A perfect agreement with IOTK exists for images $1 \mathrm{~b}, 2 \mathrm{~b}, 1 \mathrm{c}$, and $2 \mathrm{c}$.

Table 1. The correlation algorithm applied to images in Figure 3

\begin{tabular}{ccccccccc}
\hline & & & & & & & & \\
Image & $1 \mathrm{a}$ & $2 \mathrm{a}$ & $3 \mathrm{a}$ & $4 \mathrm{a}$ & $1 \mathrm{~b}$ & $2 \mathrm{~b}$ & $1 \mathrm{c}$ & $2 \mathrm{c}$ \\
$\rho$ & 0.67 & 0.67 & 0.58 & 0.55 & 0.80 & 0.94 & 0.99 & 0.98 \\
$\alpha$ & $101.25^{\circ}$ & $112.50^{\circ}$ & $112.50^{\circ}$ & $157.50^{\circ}$ & $90.00^{\circ}$ & $90.00^{\circ}$ & $90.00^{\circ}$ & $0.00^{\circ}$ \\
OST & 0.86 & 0.93 & 0.87 & 0.80 & 0.72 & 0.92 & 0.90 & 0.96 \\
$\alpha_{O S T}$ & $112.50^{\circ}$ & $101.00^{\circ}$ & $56.25^{\circ}$ & $0.00^{\circ}$ & $90.00^{\circ}$ & $45.00^{\circ}$ & $90.00^{\circ}$ & $0.00^{\circ}$ \\
\hline
\end{tabular}




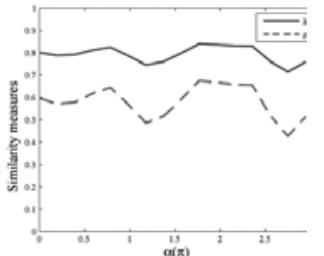

(a)

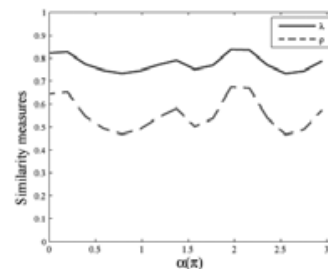

(b)

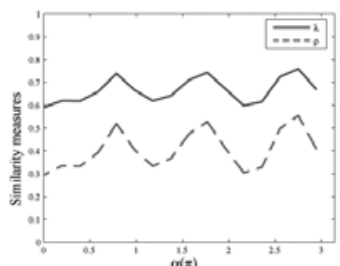

(c)

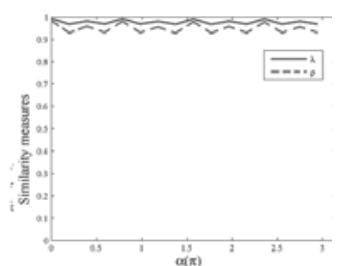

(d)

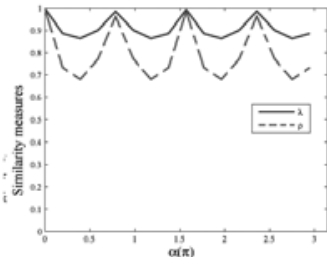

(e)

Fig. 4. Examples of correlation and similarity measures for images in Figure 3 (a) image 1a; (b) image 2a; (c) image 4a; (d) image 1c; (e) image 2c

We tested the ability of the correlation operator to exhibit circularity on the image $1 c$ by computing the mean value and the variance of $\rho$ for all $\alpha$ and the results was $(0.96,0.02)$.

\subsection{Application to Attention Focusing}

We tested the possibility of using kernel based operators to detect points of interest in complex images. Examples of such images are shown in Figures 5 a,b; they represent a famous painting by Tintoretto and a group photo under natural illuminating conditions. In both images the goal was to detect the directions of the most symmetric objects of a given size. For example in the group photo the direction of people faces.

The procedure consists in raster scanning the input image with a window, size of which is set on the basis of the human face dimensions scaled to the input frame. Inside each window kernel-based operators are computed. The algorithm returns all windows for which the value of $\lambda(\rho)$ is greater than a given threshold $\phi \in[0,1]$. Here, the threshold was set to the mean value of $\lambda(\rho)$ in all experiments. A great value of $\lambda(\rho)$ in a given direction indicates a bilateral symmetry typical of face like objects. The Figure 5 shows the results from $S K A$. Not all objects with high bilateral symmetry are faces. Nevertheless the method was able to extract all face positions, introducing an error of $17 \%$ in the evaluation of the face direction; over all experiments the percentage of not faces was $21 \%$. 


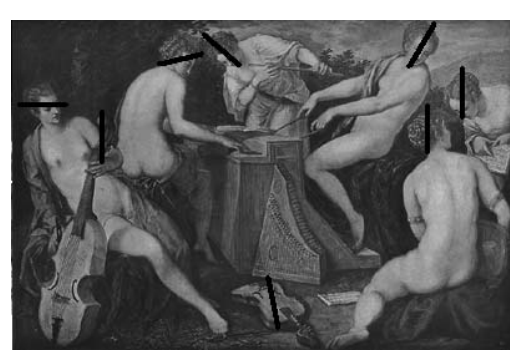

(a)

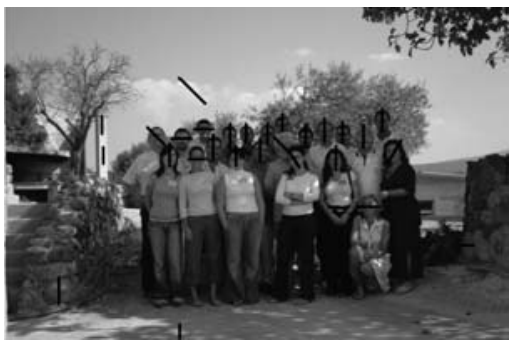

(b)

Fig. 5. Attention focusing by symmetry from $(S K A)$ : (a) Group of women (Tintoretto 1545-1588); (b) group photo

\section{Concluding Remarks}

This paper describes a new measure of axial symmetry derived from a new object feature named the "symmetry-kernel". The symmetry kernel of an object is its maximal subpart symmetric respective to a given direction. A new algorithm is derived from, based on the computation of the cross-correlation of an object with its flipped version. It is fast and not sensitive to numerical factors because computations are inner products. The algorithm was tested on both synthetic and real data. Experiments show the ability of the symmetry-kernel to detect the main directionality of an object. It has been also implemented as a local operator to detect the presence of objects in a scene and their direction. The evaluation of the distance between an object and its kernel is a crucial point and needs further investigation.

\section{References}

1. W.Khöler and H.Wallach, "Figural after-effects: an investigation of visual processes", Proc. Amer. phil. Soc., Vol.88, 269-357, 1944.

2. David O'Mara, "Automated facial metrology, chapter 4: Symmetry detection and measurement" PhD thesis, Feb. 2002

3. H.Blum and R.N.Nagel, "Shape description using weighted symmetric axis features", Pattern recognition, Vol.10, pp.167-180, 1978.

4. M.Brady, H.Asada, "Smoothed Local Symmetries and their implementation", The International Journal of Robotics Research, Vol.3, No.3, pp.36-61, 1984.

5. A. Sewisy, F. Lebert, "Detection of ellipses by finding lines of symmetry inthe images via an Hough transform applied to staright lines", Image and Vision Computing, Vol. 19 - 12, Oct. 2001, pp. 857-866

6. G.Marola, "On the detection of the axes of symmetry of symmetric and almost symmetric planar images", IEEE Trans.of PAMI, Vol.11, pp.104-108, 1989.

7. R. Manmatha, H. Sawhney, "Finding symmetry in Intensity Images", Technical Report, 1997 
8. N.Kiryati, Y.Gofman, "Detecting symmetry in grey level images (the global optimization approach)", preprint, 1997.

9. V.Di Gesù, C.Valenti, "Symmetry operators in computer vision", in Vistas in Astronomy, Pergamon, Vol.40, No.4, pp.461-468,1996.

10. V.Di Gesù, C.Valenti, "Detection of regions of interest via the Pyramid Discrete Symmetry Transform", in Advances in Computer Vision (Solina, Kropatsch, Klette and Bajcsy editors), Springer-Verlag, 1997.

11. A. D. J. Cross and E. R. Hancock, "Scale space vector fields for symmetry detection", Image and Vision Computing, Volume 17, 5-6, pp. 337-345, 1999.

12. D. Shen, H. Ip, E.K. Teoh, "An energy of assymmetry for accurate detection of global reflexion axes, Image Vision and Computing 19 (2001), pp. 283-297.

13. H. Zabrodsky, "Symmetry - A review", Technical Report 90-16, CS Dep. The Hebrew University of Jerusalem

14. V. DiGesu and B. Zavidovique, "A note on the Iterative Object Symmetry Transform", Pattern Recognition Letters, Pattern Recognition Letters, Vol. 25, pp. 1533 1545, 2004.

15. R.M. Boyton, C.L. Elworth, J. Onley, C.L. Klingberg, "Form discrimination as predicted by overlap and area", $R A D C$ - TR-60-158, 1960

16. S. Fukushima, "Division-based analysis of symmetry and its applications", IEEE PAMI, Vol. 19-2, 1997

17. D. Shen, H. Ip, K.T. Cheung, E.K. Teoh, "Symmetry detection by Generalized complex moments: a close-form solution", IEEE PAMI, Vol. 21-5, 1999

18. J. Bigun, J.M.H. DuBuf, "N-folded symmetries by complex moments in Gabor space and their application to unsupervized texture segmentation" IEEE PAMI, Vol. 16-1, 1994.

19. T. Masuda, K. Yamamoto, H. Yamada, "Detection of partial symmetyr using correlation with rotated-reflected images", Pattern Recognition, Vol. 26-8, 1993

20. D. O'Maraa, R. Owens, "Measuring bilateral symmetry in digital images". IEEE TENCON, Digital signal processing aplications, 1996

21. M. Kazhdan, B. Chazelle, D. Dobkin, A. Finkelstein, T. Funkhouser, "A reflective symmetry descriptor", $7^{\text {th }}$ ECCV, pp. 642-656, 2002

22. D.Reisfeld, H.Wolfson, Y.Yeshurun, "Detection of interest points using symmetry", 3rd IEEE ICCV Osaka, Dec. 1990.

23. Y. Bonneh, D.Reisfeld, Y.Yeshurun, "Texture discrimination by local generalized symmetry", 4th IEEE ICCV Berlin, May 1993

24. A. Merigot, B. Zavidovique, "Image analysis on massively parallel computers : An architectural point of view". J. of pattern recognition and artificial intelligence, Vol. $6 \mathrm{n}^{\circ} 2-3$, pp. 387-393, World Scientific, 1992 